\section{Driving Pressure - ein neuer/alter Parameter zur Beatmungsoptimierung?}

Bugedo $G$ et al. Driving pressure: a marker of severity, a safety limit, or a goal for mechanical ventilation. Crit Care 2017; 21: 199

Die aktuellen Leitlinien zur lungenprotektiven Beatmung beim ARDS empfehlen die Anwendung möglichst geringer, an das ideale Körpergewicht adaptierter Tidalvolumina und einen erhöhten PEEP, um die ventilatorinduzierte Lungenschädigung (VILI) zu minimieren.

Trotzdem haben Studien gezeigt, dass auch unter diesen Beatmungseinstellungen eine Lungenschädigung hervorgerufen werden kann.

Studien von Amato et al. haben gezeigt, das der „Driving-Pressure“ (Differenz aus inspiratorischem Plateaudruck [Pplat]PEEP) sehr gut mit dem Überleben nach ARDS korreliert. Weitere Studien kamen zu einem ähnlichen Ergebnis.

Bugedo und Kollegen des Departments für Intensivmedizin der Universität Santiago, Chile, haben in Critical Care hierzu in einem kurzen Review Stellung genommen.

Zunächst wird die Titration des idealen Tidalvolumens (Vt) diskutiert. Um das heute allgemein akzeptierte $\mathrm{Vt}$ zu erreichen, werden abhängig von der Grunderkrankung unterschiedlich hohe Beatmungsdrücke benötigt. Berechnet man nun den Driving Pressure (DP), wird schnell klar, dass der DP der Quotient aus Vt und der Compliance des respiratorischen Systems ist ( $\left.D P=V_{-} t / C \_R S\right)$. Je geringer der DP ist, desto geringer scheint auch die beatmungsinduzierte Schädigung der Lunge zu sein. Weiter folgern die Autoren, dass nicht die Adaptation des Vt an das ideale Körpergewicht zielführend sein könnte, sondern DP-gesteuert eine geringere Lungenschädigung analog dem Baby-Lung- Konzept von Gattinoni auftreten könnte. Das Konzept könnte durch die Erhebung des transpulmonalen DP \{Pplat-[PEEP+(Pplat ösophageal-Pösophageal endexpiratorisch)]\} durch Ösophagusdruckmessung weiter verbessert werden, da hierdurch auch extrapulmonale Faktoren wie z. B. Thoraxwandelastizität in die Berechnung einfließen.

Sucht man nun nach dem idealen DP, ist die Studienlage insgesamt uneinheitlich, sodass kein absoluter Wert für den idealen DP festgelegt werden kann. In der größten aktuellen Studie, der LUNG-SAFE-Studie, konnte bei einem DP von $>14 \mathrm{cmH}_{2} \mathrm{O}$ eine erhöhte Krankenhausmortalität gezeigt werden. Auch bei ultraprotektiver Ventilation unter ECMO konnte abhängig von den verfahrensassoziierten Risiken der Überlebungsvorteil für geringere DP gezeigt werden.

Das Rechtsherzversagen beim ARDS durch Überladung des rechten Ventrikels ist ebenfalls mit einer schlechten Prognose assoziiert. Hier scheinen Beatmungsdrücke $>30 \mathrm{cmH}_{2} \mathrm{O}, \mathrm{DP} \geq 18 \mathrm{~cm}$ $\mathrm{H}_{2} \mathrm{O}, \mathrm{PaO}_{2} / \mathrm{FiO}_{2}<150 \mathrm{mmHg}, \mathrm{paCO}_{2} \geq 48$ $\mathrm{mmHg}$ ein Rechtsherzversagen beim pneumonisch bedingten ARDS zu begünstigen.

Ein hoher DP scheint auch die diaphragmale Funktion durch Abnahme der Muskeldicke zu verschlechtern.

Nach Einschätzung der Autoren sollte der DP auch bei fehlenden prospektiven Studien und geringer Evidenz bei der Beatmungsoptimierung eingesetzt werden. Hierfür haben sie einen sehr praxisnahen Algorithmus entwickelt.
FAZIT

Der Driving Pressure ist ein wichtiger Parameter bei der Beatmungsoptimierung, den wir als Kliniker meist schon unterbewusst beachtet haben. Es gibt keinen absoluten Grenzwert; je niedriger, desto besser. Ein Abfall des DP nach PEEP-Erhöhung ist ein Hinweis auf ein Lungenrecruitment und Abfall der Schwerkräfte. Steigt nach einer PEEP-Erhöhung auch der DP an, ist ein weiteres pulmonales Recruitment nicht mehr möglich.

\section{Dr. Ralf Quabach, Solingen}

